\title{
Enquête sur la douleur et les blessures musculosquelettiques chez les optométristes canadiens
}

\author{
Kathryn Uhlman, \\ MBA, MScHQ \\ Université Queen's \\ Vlad Diaconita, MD \\ Ivey Eye Institute \\ Département d'ophtalmologie \\ Université Western
}

\author{
Alexander Mao, \\ MD, OD, MPH \\ Ivey Eye Institute \\ Département d'ophtalmologie \\ Université Western
}

\section{Rookaya Mather,} $\operatorname{MD}, \operatorname{FRCS}(\mathbf{C})$

Ivey Eye Institute

Département d'ophtalmologie

Université Western
Résumé

\section{OBJECTIF}

Accroitre notre compréhension des douleurs et des blessures musculosquelettiques professionnelles chez les optométristes canadiens.

\section{MÉTHODES}

Un sondage sur Internet à participation volontaire a été distribué à tous les membres de l'Association canadienne des optométristes. Les questions du sondage ont été adaptées à partir de la documentation pour déterminer la prévalence et l'importance des problèmes musculosquelettiques liés au travail.

\section{RÉSULTATS}

Des 121 optométristes (taux de réponse de 2,4 \%) et 169 ophtalmologistes (17\%, selon une étude antérieure) qui ont participé, $61 \%$ et $50 \%$, respectivement, ont déclaré avoir souffert de douleurs attribuables au travail au cours des 12 mois précédents $(p=0,06)$. La prévalence, l'emplacement et la gravité de la douleur étaient semblables aux constatations présentées dans les publications.

\section{CONCLUSION}

Bon nombre des professionnels des soins oculovisuels qui ont participé à notre étude ont été touchés par des douleurs musculosquelettiques liées au travail. Il faut mener d'autres recherches sur la prévention et le traitement des douleurs musculosquelettiques dans cette population.

\section{MOTS CLÉS}

Professionnel, Musculosquelettique, MS, Douleur, Lésion, Ergonomie

\section{INTRODUCTION}

Le risque de douleur musculosquelettique (MS) liée au travail chez les optométristes est désormais largement reconnu par la profession et dans les publications. ${ }^{1-3}$ Des recherches préliminaires sur les répercussions de la douleur MS liée au travail pour les professionnels des soins oculovisuels ont montré que la douleur et la gêne MS peuvent amener les professionnels de l'optométrie à chercher un autre emploi, à être hospitalisés et à solliciter un autre traitement médical. ${ }^{4}$ D'autres études ont montré que des problèmes d'ergonomie au travail pouvaient entraîner une perte de productivité, une invalidité et une retraite anticipée ${ }^{5-7}$

L’incidence des lésions liées au travail est trois fois plus élevée dans les secteurs de la santé et des services sociaux que la moyenne de l'ensemble des autres secteurs. ${ }^{8}$ Cependant, l'importance de l'exposition au risque de développer une douleur MS liée au travail varie en fonction de l'environnement de soins de santé. ${ }^{9}$ Des études récentes ont montré que la prévalence des 
douleurs musculosquelettiques liées au travail était plus élevée chez les professionnels des soins oculovisuels. À titre d'exemple, Kitzmann et ses collaborateurs ont constaté que la prévalence des douleurs musculosquelettiques était 200 à $300 \%$ plus élevée chez les ophtalmologistes que chez les médecins de famille. ${ }^{10}$

D'autres études ont révélé une prévalence de $62,1 \%$ à 82,0 \% de la douleur MS liée au travail chez les optométristes.,3 L'emplacement et la prévalence de la douleur chez les professionnels des soins oculovisuels étaient semblables dans diverses études., ${ }^{4,5,10-12}$ Les principales régions douloureuses étaient le cou ( $31,8 \%$ à $51,7 \%$ des répondants), l'épaule $(11,0 \%$ à $50,2 \%)$, le bas du dos (26,0 \% à 50,6 \%) et le haut du dos (19,0\% à 37,0\%).

Selon le Centre canadien d'hygiène et de sécurité au travail, les facteurs qui risquent d'entraîner des troubles musculosquelettiques liés au travail sont les suivants : positions du corps fixes ou contraignantes, caractère répétitif des mouvements, force concentrée sur de petites parties du corps et période de récupération trop courte entre les mouvements. ${ }^{13}$ C'est ce qui se reflète dans les résultats de l'étude de Kitzmann et ses collaborateurs, qui ont identifié plusieurs facteurs contribuant à la douleur attribuable aux troubles musculosquelettiques liés au travail chez les professionnels des soins oculovisuels. ${ }^{10}$ Ces facteurs comprenaient notamment le fait d'accomplir la même tâche de façon répétée, le travail dans des positions inconfortables ou contraignantes, le travail dans la même position pendant de longues périodes et le travail avec le torse penché vers l'avant ou dans un mouvement de torsion.

À notre connaissance, aucune étude antérieure n'a examiné la prévalence de la douleur MS liée au travail chez les optométristes au Canada. Le travail des optométristes est étroitement lié à celui des ophtalmologistes, mais les différences dans les modèles de pratique, les données démographiques et les populations de patients peuvent entraîner une différence dans les lésions musculosquelettiques. Cette étude visait à étudier la prévalence et les facteurs associés à la douleur MS liée au travail chez les optométristes canadiens et à comparer ces résultats à ceux des ophtalmologistes. ${ }^{14}$

\section{MÉTHODES}

Un sondage sur Internet à participation volontaire (Survey Monkey Inc., San Mateo, CA, É.-U.) a été distribué à tous les optométristes inscrits auprès de l'Association canadienne des optométristes (ACO) au moyen d'une liste de diffusion. Un sondage semblable a été envoyé à la Société canadienne d'ophtalmologie (SCO) pour évaluer la situation chez les ophtalmologistes canadiens. Les résultats des ophtalmologistes qui ont répondu sont publiés séparément. ${ }^{14}$ Le sondage comprenait un formulaire de consentement qui a été présenté à tous les répondants. Les questions du sondage ont été adaptées à partir de la documentation pour déterminer la prévalence et l'importance des problèmes musculosquelettiques liés au travail. Les participants n'ont reçu aucune compensation financière pour répondre au sondage. Les répondants ne pouvaient répondre au sondage qu'une seule fois à partir de leur adresse IP respective. Cette recherche a été approuvée par le Comité d'éthique de la recherche de l'Université Western.

\section{SONDAGE}

Le sondage en ligne a permis de recueillir des renseignements sur les caractéristiques démographiques et la santé personnelle des répondants, y compris l'âge, le sexe, la taille, le poids, les habitudes en matière d'exercice, les années de pratique et le nombre de patients. On a également demandé aux répondants s'ils avaient cherché à obtenir un traitement pour des douleurs ou des lésions musculosquelettiques par le passé, y compris, mais sans s'y limiter, le syndrome du canal carpien, le syndrome de De Quervain, la tendinite du biceps et l'épicondylite. Des renseignements sur le recours à la prise en charge médicale, aux options paramédicales (acupuncture, massage, physiothérapie) et à l'intervention chirurgicale ont été recueillis pour chaque diagnostic.

On a demandé à tous les répondants des groupes de l'optométrie et de l'ophtalmologie : « Au cours des 12 derniers mois, avez-vous éprouvé des douleurs musculosquelettiques que vous avez attribuées à votre travail à la clinique? ». Le sondage comprenait également des questions sur des régions douloureuses précises, notamment « cou », « épaule », « coude », « main/poignet», « haut du dos » et «bas du dos ». Pour chaque région du corps, on a demandé au répondant d'évaluer la douleur comme " aucune », « légère », « modérée » ou " grave ». La durée de la douleur a également été consignée comme « aucune », « quelques heures », « quelques jours », « quelques semaines » ou « des mois ».

Les facteurs qui ont le plus contribué à la douleur ou à la lésion musculosquelettique liée au travail ont été évalués à l'aide d'une échelle de type Likert : «NE contribue PAS », « Contribution mineure », « Contribution modérée », «Contribution majeure » et « Ne s'applique pas à ma pratique ». Les options de réponse ont été choisies en fonction d'études antérieures publiées et d'entrevues avec des fournisseurs de soins oculovisuels.

Une analyse statistique a été réalisée avec SAS (SAS Institute, Cary, Caroline du Nord, É.-U.) et Excel (Microsoft, Redmond, Washington, É.-U.) pour les variables continues et discrètes. Une analyse multivariée par régression lo- 
gistique a été réalisée pour certaines variables sur une base a priori afin de déterminer les liens avec la douleur MS.

\section{RÉSULTATS}

Cent vingt et un optométristes ont répondu au sondage, soit un taux de réponse de 2,4\%.

\section{CARACTÉRISTIQUES DES RÉPONDANTS}

Les caractéristiques de la population à l'étude sont présentées au tableau 1. Le groupe des optométristes comprenait un pourcentage plus élevé de femmes (63,6 \%) que le groupe des ophtalmologistes $(30,8 \%)$.

Les optométristes étaient légèrement plus jeunes (44,1 ans) que les ophtalmologistes ( 48,7 ans). Toutefois, le groupe des optométristes comptait plus d'années de pratique que le groupe des ophtalmologistes (21,2 ans contre 16,6 ans). La taille et l'IMC moyens étaient comparables entre les groupes. Les deux groupes faisaient de l'exercice en moyenne un peu moins de trois jours par semaine.

Lorsqu'on leur a demandé d'énumérer les diagnostics antérieurs de TMS, $62 \%$ des optométristes ont mentionné la lombalgie idiopathique, suivies du syndrome du canal carpien (24\%). La lombalgie idiopathique était aussi le diagnostic le plus fréquent chez les ophtalmologistes (31,4\%), suivie des lésions à la coiffe des rotateurs (18,9\%).

Tableau 1 : Caractéristiques des répondants

\begin{tabular}{|c|c|c|c|c|c|c|c|c|}
\hline \multirow[t]{2}{*}{ Caractéristiques } & \multicolumn{4}{|c|}{ Optometrists $(\mathrm{n}=121)$} & \multicolumn{4}{|c|}{ Ophthalmologists $(n=169)$} \\
\hline & Moyenne & ÉT & Nombre & $\%$ & Moyenne & ÉT & Nombre & $\%$ \\
\hline Âge & 44,09 & 10,63 & & & 48,73 & 13,08 & & \\
\hline Femme & & & 77 & 63,64 & & & 52 & 30,77 \\
\hline Taille & 1,71 & 0,11 & & & 1,74 & 0,09 & & \\
\hline IMC & 24,32 & 4,37 & & & 24,58 & 3,7 & & \\
\hline Jours d'exercice par semaine & 2,99 & 1,39 & & & 2,81 & 1,18 & & \\
\hline Années de pratique & 21,16 & 11,17 & & & 16,64 & 12,85 & & \\
\hline Patients par semaine & 69,47 & 28,96 & & & 139,81 & 70,26 & & \\
\hline Distance du lieu de travail $(\mathrm{km})$ & 18,87 & 20,41 & & & 11,54 & 11,17 & & \\
\hline $\begin{array}{l}\text { Transport en voiture pour se rendre } \\
\text { au travail }\end{array}$ & & & 112 & 92,56 & & & 145 & 85,8 \\
\hline \multicolumn{9}{|l|}{ Problèmes MS antérieurs } \\
\hline Syndrome de De Quervain & & & 29 & 23,97 & & & 14 & 8,28 \\
\hline Tendinite du biceps & & & 9 & 7,44 & & & 6 & 3,55 \\
\hline Épicondylite & & & 11 & 9,09 & & & 8 & 4,73 \\
\hline Sténose de la colonne cervicale & & & 15 & 12,4 & & & 17 & 10,06 \\
\hline Discopathie cervicale & & & 8 & 6,61 & & & 5 & 2,96 \\
\hline Blessure à la coiffe du rotateur & & & 10 & 8,26 & & & 23 & 13,61 \\
\hline Blessure à la coiffe du rotateur & & & 24 & 19,83 & & & 32 & 18,93 \\
\hline Épaule bloquée & & & 24 & 19,83 & & & 7 & 4,14 \\
\hline Sténose lombaire & & & 5 & 4,13 & & & 6 & 3,55 \\
\hline Discopathie lombaire & & & 9 & 7,44 & & & 15 & 8,88 \\
\hline Douleurs idiopathiques au bas du dos & & & 75 & 61,98 & & & 53 & 31,36 \\
\hline Autre & & & 15 & 12,4 & & & 24 & 14,2 \\
\hline
\end{tabular}

Données des ophtalmologistes, réf. 14 . 


\section{Douleurs musculosquelettiques liées au travail}

Soixante et un pour cent des optométristes et $50 \%$ des ophtalmologistes avaient éprouvé une douleur qui pouvait être attribuée à leur travail à la clinique au cours des 12 mois précédents $(\mathrm{p}=0,06)$. L'emplacement de la douleur, la gravité et la durée de la douleur sont comparés au tableau 2.

Chez les optométristes, la principale région douloureuse était l'épaule (41\%), suivie du bas du dos (37\%) et du cou (34\%). Malgré la prévalence élevée de la douleur à l'épaule, seulement $1 \%$ des répondants ont déclaré que cette douleur était grave; $21 \%$ ont rapporté une douleur modérée et $19 \%$ une douleur légère. La douleur dans le bas du dos était grave chez $3 \%$ des optométristes, modérée chez $10 \%$ et légère chez $24 \%$. La douleur au cou était grave chez $3 \%$ des optométristes, modérée chez $14 \%$ et légère chez $17 \%$. De même, les sites de douleur les plus courants chez les ophtalmologistes étaient le cou (46\%), le bas du dos (36\%) et l'épaule (28\%). Les deux groupes ont évalué la douleur comme étant grave, modérée ou légère pour chaque site.

Tableau 2 : Emplacement, degré et durée de la douleur

\begin{tabular}{|l|c|c|c|c|c|c|c|c|c|c|c|c|c|c|c|c|c|c|}
\hline $\begin{array}{l}\text { Emplace- } \\
\text { ment }\end{array}$ & \multicolumn{2}{|c|}{ Prévalence (\%) } & \multicolumn{3}{|c|}{ Degré de douleur (\% des répondants) } & \multicolumn{3}{|c|}{ Durée (\% des répondants) } \\
\hline & Optométristes & Ophtalmologistes & \multicolumn{2}{|c|}{ Optométristes } & \multicolumn{2}{|c|}{ Ophtalmologistes } & \multicolumn{3}{|c|}{ Optométristes } & \multicolumn{2}{|c|}{ Ophtalmologistes } \\
\hline & & & Grv & Mod & Lég & Grv & Mod & Lég & Mo & Sem & J & H & Mo & Sem & J & H \\
\hline Cou & 34 & 46 & 3 & 14 & 17 & 2 & 17 & 27 & 8 & 4 & 18 & 3 & 12 & 2 & 17 & 13 \\
\hline Épaule & 41 & 28 & 1 & 21 & 19 & 3 & 1 & 15 & 13 & 6 & 13 & 8 & 10 & 3 & 8 & 8 \\
\hline Coude & 8 & 9 & 1 & 2 & 5 & 1 & 3 & 5 & 4 & 2 & 1 & 1 & 3 & 3 & 1 & 1 \\
\hline $\begin{array}{l}\text { Main/ } \\
\text { poignet }\end{array}$ & 32 & 18 & 1 & 10 & 21 & 2 & 6 & 1 & 8 & 9 & 7 & 7 & 5 & 3 & 4 & 4 \\
\hline $\begin{array}{l}\text { Haut du } \\
\text { dos }\end{array}$ & 21 & 21 & 3 & 8 & 10 & 1 & 7 & 13 & 8 & 4 & 4 & 5 & 6 & 2 & 7 & 6 \\
\hline $\begin{array}{l}\text { Bas du } \\
\text { dos }\end{array}$ & 37 & 36 & 3 & 10 & 24 & 4 & 13 & 19 & 8 & 12 & 12 & 5 & 9 & 4 & 11 & 11 \\
\hline
\end{tabular}

Grv, Grave; Mod, Modérée; Lég, Légère Mo, Mois; Sem, semaines; J, jours; H, heures

\section{FACTEURS PROFESSIONNELS}

On a demandé aux optométristes d'évaluer plusieurs facteurs professionnels pour déterminer s'ils contribuaient de façon importante, modérée, mineure ou nulle à leur douleur ou lésion liée au travail. La plupart $(67,8 \%)$ des optométristes ont indiqué « qu'accomplir la même tâche de façon répétée » contribuait à leur douleur ou à leur lésion, suivie par «travailler dans la même position » (60,3\%) et « effectuer les examens à la lampe à fente » (55,4\%). Les facteurs considérés comme ayant contribué de façon importante à la douleur liée au travail étaient " accomplir la même tâche de façon répétée » (39,7\%), "s'étirer ou tendre les bras devant soi ou au-dessus des épaules » $(29,8 \%)$ et «travailler dans la même position pendant de longues périodes » $(17,4 \%)$. Les autres facteurs qui ont été mentionnés comme contribuant à la douleur ou à la lésion liées au travail sont énumérés au tableau 3.

Une analyse de régression logistique multivariée des données des optométristes a montré qu'un historique autodéclaré de discopathie cervicale ( $\mathrm{RC} 2,57, p=0,0312)$, de lésion de la coiffe des rotateurs ( $\mathrm{RC} 1,91, p=0,0171)$, de lombalgie idiopathique ( $\mathrm{RC} 1,79, p=0,0012)$ ou d'épaule bloquée ( $\mathrm{RC} 1,74, p=0,0099)$ était le plus souvent associé à des douleurs au cou. On a établi un lien entre la douleur à l'épaule et des antécédents autodéclarés d'épaule bloquée

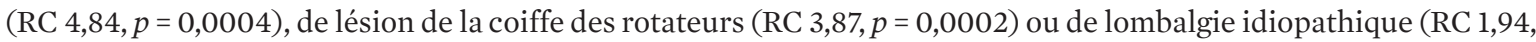

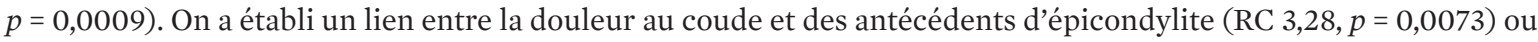
de douleur au biceps ( $\mathrm{RC} 2,84, p=0,0004)$, tandis que la douleur au poignet et à la main était associée à une lombalgie idiopathique ( $\mathrm{RC} 1,77, p=0,0005)$. On a établi un lien entre la douleur lombaire et la lombalgie idiopathique (RC 2,38, $p<0,0001$ ) (tableau 4). 
Tableau 3 : Facteurs contribuant aux douleurs musculosquelettiques liées au travail chez les optométristes

\begin{tabular}{|c|c|c|c|}
\hline \multirow[t]{2}{*}{ Facteur } & \multicolumn{3}{|c|}{$\begin{array}{l}\text { Degré de contribution } \\
\text { (\% des répondants) }\end{array}$} \\
\hline & Aucune & $\begin{array}{l}\text { Moderate } \\
\text { or Major }\end{array}$ & Major \\
\hline Accomplir la même tâche de façon répétée & 67,77 & 57,02 & 39,67 \\
\hline Travailler dans la même position pendant de longues périodes & 60,33 & 39,67 & 17,36 \\
\hline S'étirer ou tendre les bras devant soi ou au-dessus des épaules & 54,55 & 42,98 & 29,75 \\
\hline Travailler dans des positions inconfortables ou contraignantes & 53,72 & 30,58 & 8,26 \\
\hline Continuer de travailler en cas de blessure ou de maladie & 50,41 & 31,40 & 9,92 \\
\hline Pauses insuffisantes pendant la journée & 51,24 & 28,93 & 9,92 \\
\hline $\begin{array}{l}\text { Travailler avec le torse penché vers l'avant ou dans un mouvement } \\
\text { de torsion }\end{array}$ & 47,93 & 28,93 & 12,40 \\
\hline $\begin{array}{l}\text { Travailler de longues heures ou avoir des heures de travail } \\
\text { irrégulières }\end{array}$ & 44,63 & 24,79 & 9,09 \\
\hline $\begin{array}{l}\text { Travailler avec la tête penchée vers l'avant ou dans un mouvement } \\
\text { de torsion. }\end{array}$ & 44,63 & 23,14 & 9,92 \\
\hline Presbytie/erreur de réfraction & 42,15 & 22,31 & 13,22 \\
\hline Effectuer les examens à la lampe à fente & 55,37 & 27,27 & 12,40 \\
\hline Examen indirect du fond de l'œil & 50,41 & 19,83 & 6,61 \\
\hline
\end{tabular}

Tableau 4 : Régression logistique multiple chez les optométristes

\begin{tabular}{|c|c|c|c|c|c|}
\hline $\begin{array}{l}\text { Type de douleurs } \\
\text { musculosquelettiques } \\
\text { liées au travail }\end{array}$ & Association & $\begin{array}{l}\text { Rapport de } \\
\text { cotes }\end{array}$ & IC & Valeur $\mathbf{p}$ & $\mathbf{N}$ \\
\hline \multirow{5}{*}{ Douleur au cou } & Discopathie cervicale & 2,57 & $(1,089-6,049)$ & 0,0312 & \multirow{5}{*}{41} \\
\hline & Âge & 0,95 & $(0,905-0,992)$ & 0,0202 & \\
\hline & Blessure à la coiffe du rotateur & 1,91 & $(1,122-3,239)$ & 0,0171 & \\
\hline & Épaule bloquée & 1,74 & $(1,143-2,660)$ & 0,0099 & \\
\hline & Douleur lombaire idiopathique & 1,79 & $(1,259-2,545)$ & 0,0012 & \\
\hline \multirow{4}{*}{ Douleur à l'épaule } & Âge & 0,93 & $(0,885-0,978)$ & 0,0046 & \multirow{4}{*}{71} \\
\hline & Blessure à la coiffe du rotateur & 3,87 & $(1,896-7,907)$ & 0,0002 & \\
\hline & Épaule bloquée & 4,84 & $(2,014-11,616)$ & 0,0004 & \\
\hline & Douleur lombaire idiopathique & 1,94 & $(1,310-2,873)$ & 0,0009 & \\
\hline \multirow{2}{*}{ Douleur au coude } & Épicondylite & 3,28 & $(1,690-6,369)$ & 0,0004 & \multirow{2}{*}{111} \\
\hline & Douleur au biceps & 2,84 & $(1,325-6,082)$ & 0,0073 & \\
\hline $\begin{array}{l}\text { Douleur à la main ou au } \\
\text { poignet }\end{array}$ & Douleur lombaire idiopathique & 1,77 & $(1,282-2,452)$ & 0,0005 & 83 \\
\hline Douleur au haut du dos & Âge & 0,93 & $(0,876-0,977)$ & 0,0051 & 95 \\
\hline Douleur au bas du dos & Douleur lombaire idiopathique & 2,38 & $(1,684-3,369)$ & $<0,0001$ & 75 \\
\hline
\end{tabular}


DISCUSSION

Dans cette étude, $61 \%$ des optométristes avaient éprouvé des douleurs musculosquelettiques attribuables à leur travail au cours des 12 mois précédents, comparativement à $50 \%$ des ophtalmologistes. Ces résultats sont semblables à ceux déclarés par Gromacki et ses collaborateurs : 62,1\% des optométristes ont éprouvé des douleurs musculosquelettiques tout au long de leur carrière. ${ }^{3}$ Toutefois, nous avons observé une prévalence plus faible de la douleur MS chez les optométristes que celle qui a été rapportée dans une étude précédente menée par Long et son équipe. ${ }^{4}$ Long a constaté que $82 \%$ des optométristes ont signalé une gêne physique liée au travail au cours des 12 derniers mois. Ce pourcentage plus élevé de répondants signalant une gêne physique pourrait être attribué à l'utilisation du terme "gêne », qui comprend probablement un plus large éventail de problèmes musculosquelettiques que la « douleur » dans l'esprit des répondants. Nos résultats se rapprochent davantage de ceux d'autres études sur les douleurs MS chez les professionnels des soins oculovisuels, y compris ceux de Hyer et coll. ${ }^{11}$ (prévalence de 62,4 \%), Al-Marwani Al-Juhani et coll. ${ }^{15}$ (72 \%) et Kitzmann et coll. ${ }^{10}$ (11 à $46 \%$ selon la région du corps).

Bien qu'aucune autre étude n’ait directement comparé la prévalence de la douleur MS chez les optométristes et les ophtalmologistes, Al-Marwani Al-Juhani et coll..$^{15}$ ont comparé la prévalence de la douleur au cou et au dos chez les ophtalmologistes et d'autres fournisseurs de soins oculovisuels, y compris un petit sous-ensemble d'optométristes (11 des 165 répondants). Ils ont constaté des pourcentages plus élevés de douleur chez les optométristes (82\%) et chez tous les autres fournisseurs de soins oculovisuels (72\%) que chez les ophtalmologistes (67\%). Cela est conforme à nos constatations.

Bien qu'il soit possible de comparer les taux de gêne et de douleur MS entre les études mentionnées, les résultats ne sont pas directement comparables, car les périodes de temps pendant lesquelles la douleur a été ressentie ne sont pas les mêmes dans les deux études. Certaines études, comme celle d'Al-Marwani Al-Juhani et coll. ${ }^{15}$ demandaient aux répondants s'ils avaient ressenti des douleurs musculosquelettiques liées au travail sans préciser de durée. ${ }^{4}$ Dans l'étude de Kitzmann et coll. ${ }^{10}$ la fenêtre de temps était étroite (les 30 derniers jours), ce qui peut avoir réduit le pourcentage de répondants ayant signalé une douleur.

L’âge moyen des optométristes qui ont participé à notre étude (44,1 ans) est comparable à celui des participants à d'autres études. ${ }^{4,10,11}$ Il n'y avait pas de lien entre l'âge accru et la douleur MS chez nos répondants.

Dans l'étude menée par Long et coll.4, les optométristes signalaient une gêne le plus souvent dans le cou (51,7 \%), l'épaule (50,2 \%) et le bas du dos (45,9\%). Ces trois sites avaient également la prévalence la plus élevée de la douleur dans notre étude, bien qu'avec un classement différent (41 \% à l'épaule, $37 \%$ au bas du dos et $34 \%$ au cou). De même, les sites de douleur les plus fréquemment signalés par les ophtalmologistes dans notre étude étaient le cou (46\%), le bas du dos (36\%) et l'épaule (28\%).

Al-Marwani Al-Juhani et coll. ${ }^{15}$ se sont penchés sur la gravité de la gêne et de la douleur MS liées au travail. Parmi les professionnels des soins oculovisuels qui avaient éprouvé des douleurs musculosquelettiques liées au travail, la douleur était grave dans $10 \%$ des cas, modérée dans $34 \%$ des cas et légère dans $53 \%$ des cas. Dans notre étude, la plupart des optométristes qui ont déclaré avoir éprouvé des douleurs à l'épaule liées au travail (53,7 \%) ont déclaré que la douleur était de modérée à grave.

Al-Marwani Al-Juhani et coll. ${ }^{15}$ ont également constaté que la prévalence des maux de dos était 23,4 \% plus faible chez les personnes qui faisaient de l'exercice au moins trois fois par semaine, comparativement à celles qui ne faisaient pas d'exercice. Long et coll. ${ }^{4}$ ont constaté que les femmes étaient plus susceptibles d'éprouver une gêne MS liée au travail que les hommes. Il n'y avait pas de lien entre le sexe et la douleur MS chez nos répondants.

\section{FACTEURS PROFESSIONNELS}

En examinant les facteurs professionnels qui ont contribué aux douleurs musculosquelettiques liées au travail chez les ophtalmologistes, Kitzmann et coll. ${ }^{10}$ ont constaté que les trois facteurs les plus fréquemment cités étaient « travailler dans des positions inconfortables ou contraignantes » $(57 \%)$, «travailler dans la même position pendant de longues périodes » $(55 \%)$ et «accomplir la même tâche de façon répétée » (45\%). Nous avons obtenu des résultats semblables chez les optométristes : les trois facteurs les plus fréquemment cités étaient « accomplir la même tâche de façon répétée » $(67,8 \%)$, «travailler dans la même position pendant de longues périodes » $(60,3 \%)$ et « effectuer 
les examens à la lampe à fente » (55,4\%). Dans notre étude précédente chez les ophtalmologistes, les causes les plus courantes de douleur liée au travail étaient « accomplir la même tâche de façon répétée » (58,6 \%), «travail dans des positions inconfortables ou contraignantes » $(52,1 \%)$ et « travailler de longues heures ou avoir des heures de travail irrégulières » $(50,3 \%)$.

\section{LIMITES DE L’ÉTUDE}

Les résultats actuels sont limités par le faible taux de réponse des optométristes $(2,4 \%)$, ce qui soulève la question de savoir si les résultats reflètent fidèlement la population étudiée. Un biais de réponse peut être une autre limitation, puisque les personnes souffrant de douleur peuvent avoir été plus susceptibles de répondre au sondage que la population en général. De plus, alors que seulement 52,9\% des optométristes au Canada sont des femmes, ${ }^{16}$ celles-ci représentaient $63,6 \%$ de nos répondants. Enfin, notre questionnaire est vulnérable à la fois à un biais de récence et à un biais de gravité, étant donné que les répondants sont plus susceptibles de se souvenir des lésions les plus récentes et les plus graves.

\section{HEURES DE TRAVAIL PERDUES}

Il est important de comprendre les caractéristiques des douleurs musculosquelettiques liées au travail chez les professionnels des soins oculovisuels, car il a été démontré qu'une telle douleur a des répercussions sur leur carrière. Les douleurs musculosquelettiques liées au travail peuvent entraîner une perte de travail. Dans une étude portant sur des chirurgiens orthopédiques pédiatriques, $51 \%$ des personnes comptant de 21 à 30 ans de carrière avaient pris un congé du travail en raison de lésions MS. ${ }^{17}$ Dans une étude menée auprès d'optométristes qui avaient éprouvé une gêne liée au travail, 32 \% avaient cessé de travailler en raison de la douleur et $4 \%$ des personnes interrogées ont déclaré avoir complètement cessé de travailler en tant qu'optométristes. ${ }^{1}$ Une autre étude a révélé que des problèmes d'ergonomie au travail entraînent une faible productivité au travail et conduisent à l'invalidité et à la retraite anticipée. ${ }^{5}$

\section{EXERCICE ET ENTRAÎNEMENT SPORTIF}

D'autres recherches sont nécessaires pour définir les pratiques exemplaires en matière de prévention et de traitement de la douleur MS chez les professionnels des soins oculovisuels. Une recension des écrits a fait ressortir des preuves modérées selon lesquelles l'entraînement physique pourrait améliorer la force et l'endurance, et donc avoir des effets positifs sur les résultats MS chez les professionnels de la santé ${ }^{18}$ Une étude réalisée auprès de dentistes aux Pays-Bas a suggéré que la mise en œuvre de recommandations pour une posture appropriée liée au travail avait permis d'améliorer les troubles musculosquelettiques (TMS) : 84 \% des personnes interrogées ont déclaré qu'elles avaient fait diminuer leurs TMS ou les avaient fait disparaitre. ${ }^{19} \mathrm{D}$ 'autres interventions préventives, notamment l'optimisation des instruments et des dispositifs actuels, et une éducation adéquate pourraient limiter la prévalence de la douleur MS chez les professionnels des soins oculovisuels. ${ }^{20}$

\section{CONCLUSION}

En conclusion, les résultats de notre étude concordaient avec ceux d'autres publications portant sur la douleur MS chez les professionnels des soins oculovisuels. Parmi les optométristes qui ont participé au sondage, $61 \%$ avaient éprouvé des douleurs musculosquelettiques attribuables au travail au cours des 12 mois précédents. Les régions de douleur les plus courantes étaient l'épaule, le bas du dos et le cou. La prévalence, l'emplacement et la gravité de la douleur étaient semblables à ceux qui ont été rapportés dans les publications. Il faut mener d'autres recherches sur la prévention et le traitement de la douleur MS liée au travail chez les professionnels des soins oculovisuels en raison de son effet nuisible sur la carrière. 


\section{RÉFÉRENCES}

Long J., Burgess-Limerick R., Stapleton F. Personal consequences of work-related physical discomfort : An exploratory study. Clin Exp Optom 2014;97(1):30-5.

Newman B.. Ergonomics and the optometrist. Optometry 2005;76(2):77.

Gromacki, S., Wicker, D., Leung, H. Ergonomics in eye care. Optom Vis Sci 2002;79 (Supplement):101.

Long, J., Naduvilath, T.J., Hao, L. et coll. Risk factors for physical discomfort in Australian optometrists. Optom Vis Sci 2011;88(2):317-26.

Honavar S. Head up, heels down, posture perfect : Ergonomics for an ophthalmologist. Indian J Ophthalmol 2017;65(8):647.

Soueid A., Oudit D., Thiagarajah S., Laitung G. The pain of surgery : Pain experienced by surgeons while operating. Int J Surg 2010;8(2):11820.

Sivak-Callcott J.A., Mancinelli C.A., Nimbarte A.D. Cervical occupational hazards in ophthalmic plastic surgery. Curr Opin Ophthalmol 2015 Jul;26(5):392-8.

L'Association des commissions des accidents du travail du Canada. Statistiques nationales sur les accidents du travail, les maladies et les décès (Rep.) 2016.

Waters T., Collins J., Galinsky T., Caruso C. NIOSH research efforts to prevent musculoskeletal disorders in the health industry. Orthop Nurs 2006 Nov-Dec;25(6):380-9.

Kitzmann A.S., Fethke N.B., Baratz K.H., Zimmerman M.B., Hackbarth D.J., Gehrs K.M. A survey study of musculoskeletal disorders among eye care physicians compared with family medicine physicians. Ophthalmology 2012;119(2):213-20.

Hyer, J.N., Lee, R.M., Chowdhury, H.H., Smith, H.B., Dhital, A. Khandwala, M. National survey of back \& neck pain among consultant ophthalmologists in the United Kingdom. Int Ophthalmol 2015;35(6):769-75.
Marx, J.L., Wertz, F.D., Dhimitri, K.C. Work-related musculoskeletal disorders in ophthalmologists. Tech Ophthalmol 2005;3(1), 54-61.

Centre canadien d'hygiène et de sécurité au travail. Troubles musculosquelettiques liés au travail (TMSLT) : Réponses SST. 11 mai 2018. Extrait de https://www.cchst.ca/oshanswers/diseases/rmirsi.html

Diaconita V., Uhlman K., Mao A., Mather R. Survey of occupational musculoskeletal pain and injury in Canadian ophthalmology. Can $J$ Ophthalmol 2018; sous presse.

Al-Marwani Al-Juhani M., Khandekar R., Al-Harby M., Al-Hassan A. Edward D.P. Neck and upper back pain among eye care professionals. Occup Med (Lond) 2015 Dec;65(9):753-7.

L'Institut canadien d'information sur la santé Les dispensateurs de soins de santé au Canada : Profils provinciaux, 2007 à 2016 - Tableaux de données(Rep.) 2016.

Alzahrani M.M., Alqahtani S.M., Tanzer M., Hamdy R.C. Musculoskeletal disorders among orthopedic pediatric surgeons: An overlooked entity. $J$ Child Orthop 2016 Oct;10(5):461-6.

Tullar, J.M., Brewer, S., Amick, B.C., et coll. Occupational safety and health interventions to reduce musculoskeletal symptoms in the health care sector. J Occup Rehabil 2010 Jun;20(2):199-219.

Lavender, S.A., Sommerich, C.M., Johnson, M.R., Radin, Z. Developing ergonomic interventions to reduce musculoskeletal disorders in grocery distribution centers. Proc Hum Factors Ergon Soc Annu Meet 2010;54(15):1229-33.

Marx J.L. Ergonomics: Back to the future. Ophthalmology 2012;119(2):211-2. 